\title{
OCORRÊNCIA DE DISFUNÇÃO TEMPOROMANDIBULAR (DTM) E SUA RELAÇÃO COM HÁBITOS ORAIS DELETÉRIOS EM CRIANÇAS DO MUNICÍPIO DE MONTE NEGRO - RO
}

\section{Occurrence of temporomandibular disorder (TMD) and its relationship with harmful oral habits in children from Monte Negro - RO}

\author{
Luciana Biral Mendes Merighi ${ }^{(1)}$, Marcela Maria Alves da Silva ${ }^{(2)}$, Amanda Tragueta Ferreira ${ }^{(3)}$, \\ Katia Flores Genaro ${ }^{(4)}$, Giédre Berretin-Felix ${ }^{(5)}$
}

\begin{abstract}
RESUMO
Objetivo: verificar a ocorrência de disfunção temporomandibular em crianças do município de Monte Negro/RO, correlacionando os achados à presença de hábitos orais deletérios. Métodos: investigouse a presença de quadros de disfunção temporomandibular, classificando-se em disfunção temporomandibular articular, muscular ou mista, bem como a presença de hábitos orais deletérios de sucção (mamadeira, chupeta e digital) e mastigação (onicofagia, morder objetos, morder a mucosa oral ou labial, bruxismo e apertamento dentário), em 79 crianças de seis a 11 anos (41 meninas e 38 meninos). Resultados: dentre as 79 crianças investigadas, $27(34,18 \%)$ apresentaram sinais de disfunção temporomandibular, sendo a disfunção articular a mais encontrada $(48,15 \% \mathrm{n}=13)$, seguida da mista $(33,33 \%, n=9)$ e, com menor ocorrência, a muscular $(18,52 \%, n=5$ crianças). Hábitos orais deletérios foram encontrados em $69,62 \%(n=55)$ da amostra, sendo maior o número de crianças com hábitos de mastigação $(39,91 \%, n=26)$ que de sucção $(8,86 \%, n=7)$, observando-se casos em que os dois tipos de hábito estavam presentes $(27,85 \%, n=22)$. Os resultados não demonstraram associação entre a presença de hábitos orais deletérios e disfunção temporomandibular (teste Qui-quadrado), independentemente do tipo de hábito. Conclusão: das crianças avaliadas, um terço (34\%) apresentou sinais de disfunção temporomandibular, não havendo associação entre a disfunção temporomandibular com a presença de hábitos orais deletérios.
\end{abstract}

DESCRITORES: Articulação Temporomandibular; Criança; Hábitos

(1) Fonoaudióloga; Clínica Long Life; Mestre em Fonoaudiologia pela Faculdade de Odontologia de Bauru da Universidade de São Paulo.

(2) Fonoaudióloga; Mestranda do Programa de Pós-Graduação Interunidades em Bioengenharia da Universidade de São Paulo, Campus São Carlos.

(3) Fonoaudióloga; Aluna da Prática Profissionalizante em Linguagem Infantil da Clínica de Fonoaudiologia da Faculdade de Odontologia de Bauru da Universidade de São Paulo.

(4) Fonoaudióloga; Professora Associada do Departamento de Fonoaudiologia da Faculdade de Odontologia de Bauru da Universidade de São Paulo; Livre-docente pela Faculdade de Odontologia de Bauru da Universidade de São Paulo.

(5) Fonoaudióloga; Professora Doutora do Departamento de Fonoaudiologia da Faculdade de Odontologia de Bauru da Universidade de São Paulo; Doutora em Fisiopatologia em Clínica Médica pela Faculdade de Medicina de Botucatu da Universidade Estatual Paulista "Júlio de Mesquita Filho".

\section{INTRODUÇÃO}

A articulação temporomandibular (ATM) é considerada a mais complexa das articulações do corpo humano ${ }^{1}$. É composta de estruturas ósseas, cartilaginosas, ligamentos e musculatura associada ${ }^{2,3}$, sendo responsável pelos movimentos mandibulares, em decorrência das ações dos músculos mastigatórios ${ }^{4,5}$.

A ocorrência de situações desfavoráveis que afetam a ATM é freqüente, pois essa articulação precisa acomodar adaptações oclusais, musculares e cervicais ${ }^{3}$. Assim, condições de desequilíbrio podem resultar em quadros de disfunção da ATM, que corresponde ao termo genérico relacionado a um conjunto clínico de sinais e sintomas envolvendo os músculos mastigatórios, a própria articu- 
lação e estruturas associadas, denominado disfunção temporomandibular (DTM) ${ }^{6,7}$.

A prevalência de DTM é maior no sexo feminino e na faixa etária entre 21 e 40 anos ${ }^{8-11}$, tendo sido descrita como uma condição que afeta, principalmente, adultos. Estudos epidemiológicos têm relatado baixa ocorrência de sinais e sintomas de DTM em crianças até os seis anos ${ }^{12}$, aumentando após essa idade até a adolescência, verificando-se índices semelhantes aos dos adultos ${ }^{13,14}$.

Quanto à etiologia das DTMs, esta é multifatorial, associando-se fatores predisponentes, iniciadores e perpetuantes ${ }^{15,16}$, incluindo distúrbios da oclusão, das bases ósseas maxilar e mandibular, fatores traumáticos, problemas degenerativos, alterações musculares como hiperatividade ou hipoatividade, modificações funcionais e hábitos nocivos que levam à sobrecarga persistente na ATM ou na musculatura, estresse e problemas emocionais $2,3,17$.

De acordo com a literatura, há relação significante entre hábitos orais deletérios de sucção digital, morder as bochechas, onicofagia e bruxismo com a presença de sinais e/ou sintomas de DTM em crianças ${ }^{18-20}$, além de quadros de deglutição atípica, também associados a esta disfunção ${ }^{21}$.

Estudos sobre DTM em crianças são recentes e têm demonstrado a importância da detecção e intervenção, buscando reduzir os possíveis prejuízos relacionados, além de reforçar a importância de reconhecer crianças com pré-disposição para desordens do sistema estomatognático ${ }^{22}$.

Vale ressaltar que, na literatura, há escassez de estudos que descrevem tais características, principalmente na população da região de Rondônia, dificultando propostas de reabilitação e orientação junto àquela população. Sendo assim, o objetivo deste estudo foi verificar a ocorrência de DTM em crianças de um município de Rondônia/Brasil, correlacionando os achados à presença de hábitos orais deletérios.

\section{MÉTODOS}

Para a seleção dos sujeitos dessa pesquisa, foi analisada a lista de matrícula dos alunos de uma escola da zona urbana do município de Monte Negro/RO. Os pais ou responsáveis pelas crianças foram convocados para esclarecimentos sobre a pesquisa e realização de entrevista fonoaudiológica. A partir dos dados obtidos foram excluídas aquelas que apresentavam quadros neurológicos, sindrômicos, malformações craniofaciais ou cuja faixa etária não atendia à proposta da pesquisa. Assim, foram selecionadas, aleatoriamente, 79 crianças na faixa etária de seis a 11 anos, sendo 41 do sexo feminino e 38 do masculino.
Durante a entrevista realizada com os pais foi investigada a presença dos hábitos orais deletérios de sucção e os mastigatórios.

Para os hábitos de sucção investigou-se o uso de mamadeira, a sucção de chupeta e digital, considerando-se a presença e a duração dos mesmos, tendo em vista a possibilidade de acarretar efeito negativo sobre o equilíbrio do sistema estomatognático. Assim, foram computadas aquelas que referiram apresentar hábitos na época da entrevista ou com histórico de hábito persistente após os 3 anos de idade ${ }^{23-25}$. Foram considerados hábitos mastigatórios a onicofagia, morder objetos, morder a mucosa oral, bruxismo (ranger de dentes noturno) e apertamento dentário, relacionados ao momento atual. Os dados obtidos foram utilizados para classificação (hábitos de sucção e hábitos mastigatórios), não sendo considerada a análise dos diferentes tipos de hábitos isoladamente.

Para a investigação e diagnóstico da DTM realizou-se exame clínico seguindo-se o Protocolo de Avaliação do Sistema Estomatognático utilizado na Clínica de Fonoaudiologia da Faculdade de Odontologia de Bauru/Universidade de São Paulo, constituído por inspeção intra e extra-oral, palpação dos músculos: trapézio, esternocleidomastóideo, temporal e masseter e pterigóideos mediais. Também foi realizada a palpação das ATMs e análise da movimentação mandibular, com utilização de régua milimetrada para medidas de máxima abertura oral e estetoscópio para a verificação de ruídos articulares.

A partir dos achados clínicos, as crianças foram classificadas segundo os critérios da Academia Americana de Dor Orofacial ${ }^{2}$.

- Grupo com DTM Muscular - relato de dor na região dos músculos mastigatórios durante exame funcional e/ou palpação muscular; exclusão dos casos com sinais de DTM artrogênica.

- Grupo com DTM Articular - presença de deslocamento de disco, limitação de máxima abertura oral (menor ou igual a $35 \mathrm{~mm}$ ), deflexão ou desvios mandibulares, dor espontânea e/ou à palpação na ATM; exclusão dos casos com sinais de DTM miogênica.

- Grupo com DTM Misto - presença de sinais de DTM muscular e articular.

A pesquisa foi aprovada pelo Comitê de Ética em Pesquisa da Instituição, sob número 121/2005, respeitando todos os aspectos éticos necessários para pesquisas dessa natureza. 
Os dados foram tabulados em banco de dados e submetidos à análise estatística utilizando o Teste Qui-quadrado, tendo-se considerado o nível de 5\% de significância $(p<0,05)$.

\section{RESULTADOS}

Do total de 79 crianças avaliadas, 52 delas $(65,82 \%)$ não apresentaram sinal e/ou sintoma de DTM, enquanto 27 crianças $(34,18 \%)$ apresentaram quadro de DTM, com maior ocorrência no sexo feminino (Figura 1). Dentre estas 27, a Disfunção Articular foi a mais encontrada, seguida da DTM Mista (articular e muscular) e, em menor ocorrência, a DTM Muscular (Figura 2).

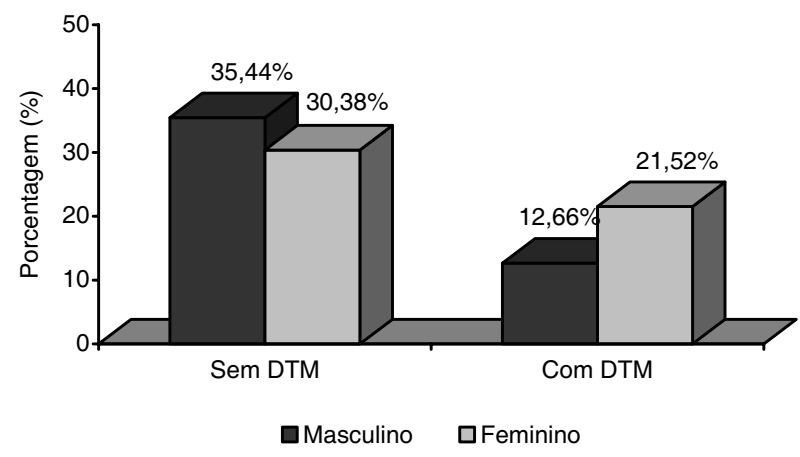

Figura 1 - Distribuição da amostra de acordo com o sexo e a ocorrência de DTM

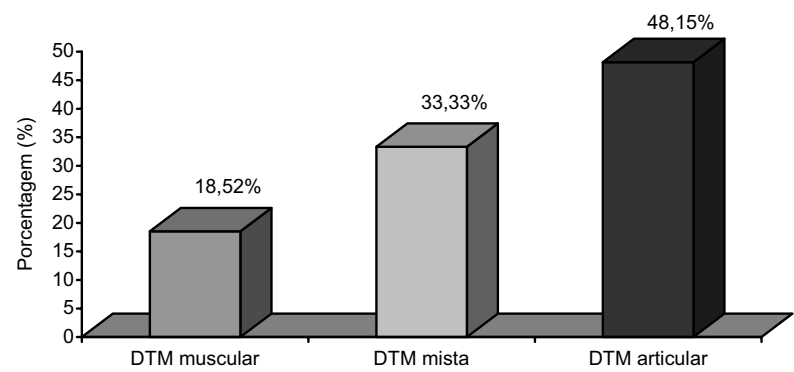

Figura 2 - Ocorrência dos diferentes tipos de DTM na amostra avaliada

Em relação à investigação dos hábitos orais deletérios, das 79 crianças avaliadas, mais da metade $(69,62 \%, n=55)$ apresentou hábitos orais, com maior ocorrência dos mastigatórios $(39,91 \%$, $\mathrm{n}=26)$ em relação aos de sucção $(8,86 \%, n=7)$, sendo também observados casos em que os dois tipos estavam presentes $(27,85 \%, n=22)$, conforme visualizado na Figura 3.

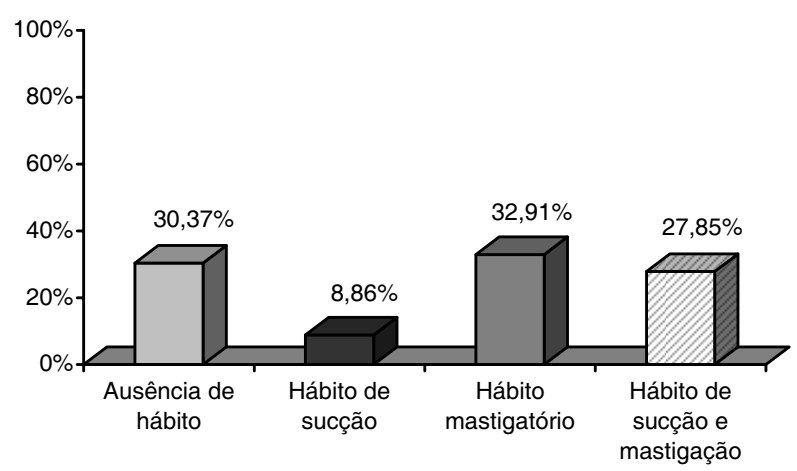

Figura 3 - Distribuição da amostra de acordo com a ocorrência de hábitos orais deletérios

A coexistência de DTM e hábitos orais foi apresentada por 16 crianças $(20,25 \%)$. A associação da DTM com os tipos de hábitos foi encontrada em 9 crianças $(11,39 \%)$ com o hábito de sucção. Com relação ao mastigatório, este esteve presente em 14 sujeitos (17,72\%), não havendo associação entre a presença de hábitos orais deletérios e DTM, independente do tipo de hábito (Tabela 1).

\section{DISCUSSÃO}

A ocorrência de quadros de DTM e hábitos orais deletérios, principalmente na infância, interferem na funcionalidade do sistema estomatognático, podendo resultar em alterações na respiração, mastigação, deglutição e fala ${ }^{12,15,26}$. Nesse sentido, o presente estudo buscou relacionar tais achados em crianças residentes no município de Monte Negro, do Estado de Rondônia-Brasil.

Aproximadamente um terço das crianças avaliadas nessa pesquisa apresentaram sinais de DTM, corroborando o estudo que verificou quadros de DTM em $30 \%$ da amostra com idade entre sete e 13 anos ${ }^{27}$. Alguns autores também encontraram porcentagem semelhante de ocorrência de DTM, porém estes estudaram crianças na faixa etária de três a cinco anos ${ }^{21,28}$. Da mesma forma, em outro estudo, foi verificado que $35 \%$ dos casos com idade variando entre seis e 16 anos apresentavam, pelo menos, um sinal de DTM ${ }^{29}$.

A partir da classificação da DTM, segundo a Academia Americana de Dor Orofacial ${ }^{2}$, verificouse maior ocorrência de DTM articular em relação a DTM mista e muscular. Não foram encontrados na literatura trabalhos que tenham utilizado tal classificação na infância e adolescência. Entretanto, um estudo com crianças entre seis e 12 anos, verificou elevada ocorrência de dor muscular à palpação, principalmente no músculo pterigóideo 
Tabela 1 - Ocorrência de DTM e hábitos orais deletérios, e resultado do teste de associação

\begin{tabular}{lllll}
\hline \multirow{2}{*}{ Hábitos orais } & & \multicolumn{2}{c}{ DTM } & \multirow{2}{*}{ Valor de “p” } \\
\cline { 3 - 4 } & & Ausente & Presente & \\
\hline \multirow{2}{*}{ Sucção } & Ausente & $13(17 \%)$ & $11(14 \%)$ & \\
& Presente & $39(49 \%)$ & $16(20 \%)$ & $0,1490(\mathrm{NS})$ \\
\hline \multirow{2}{*}{ Mastigatório } & Ausente & $32(40 \%)$ & $18(23 \%)$ & \\
& Presente & $20(25 \%)$ & $9(12 \%)$ & 0,6537 (NS) \\
\cline { 3 - 4 } & Ausente & $18(23 \%)$ & $13(16 \%)$ & \\
\hline
\end{tabular}

Teste Qui-quadrado

lateral $(80,1 \%)$, enquanto os sinais de ruído articular foram encontrados para $35,3 \%$ da amostra estudada ${ }^{30}$.

Disfunções articulares decorrem de possíveis microtraumas crônicos, provocados por hábitos orais deletérios relacionados à função mastigatória e movimentos excessivos da mandíbula, além de macrotraumas ${ }^{17}$. Ressalta-se que, na maior parte das crianças avaliadas, com e sem DTM, foi encontrada presença de hábitos orais deletérios. Além disso, alterações dento-oclusais, freqüentes na população local e não incluídas neste estudo, podem ocasionar hiperatividade muscular crônica, sendo esta considerada fator etiológico associado a problemas articulares ${ }^{31}$.

No que diz respeito ao sexo, as meninas apresentaram maior ocorrência de DTM que os meninos, corroborando pesquisas realizadas com adolescentes ${ }^{8-10,14}$, as quais também consideraram a influência do tratamento ortodôntico ${ }^{11}$. Porém, outros estudos demonstram não haver maior prevalência de DTM no sexo feminino na infância e na adolescência ${ }^{7,32}$.

Relacionada à presença de hábitos orais deletérios, neste trabalho foi verificado que $69,62 \%$ das crianças entre seis a 11 anos apresentaram algum tipo de hábito, o que vem ao encontro com os achados da literatura, que apontam alta ocorrência de hábitos orais deletérios (sucção não-nutritiva, nutritiva e a digital, onicofagia e bruxismo) em crianças de quatro a seis anos, da cidade de Manaus ${ }^{33}$. Outro estudo com crianças de quatro anos de idade, também verificou presença desses hábitos em $95 \%$ dos casos $^{23}$; e uma pesquisa realizada na Universidade Federal de Santa Maria/RS, com crianças de cinco a 14 anos, constatou que $73 \%$ apresentavam hábitos orais deletérios ${ }^{34}$

Ao comparar a ocorrência dos diferentes hábitos investigados, foi observado predomínio do hábito mastigatório em relação ao de sucção, concordando com o estudo em que a onicofagia foi o hábito mais encontrado em relação aos hábitos de sucção digital e bruxismo ${ }^{35}$. Por outro lado, outros autores observaram maior presença do hábito de sucção que o mastigatório, discordando dos achados desta pesquisa ${ }^{20,23}$.

A análise estatística desta pesquisa não mostrou associação entre a presença de hábitos de sucção e mastigatórios aos quadros de DTM, corroborando o estudo em que, dentre os hábitos investigados, apenas a deglutição atípica estava relacionada à ocorrência da DTM ${ }^{21}$, sendo necessário ressaltar que não foram investigadas questões voltadas à deglutição neste estudo.

Outros aspectos, tais como padrão mastigatório, condição oclusal e tipologia facial, não considerados neste trabalho, poderiam estar relacionados à presença de DTM ${ }^{18}$, uma vez que autores verificaram que indivíduos com padrão mastigatório unilateral podem apresentar alterações musculares e transtornos da ATM ${ }^{36}$, principalmente devido a interferências oclusais ${ }^{6,27,30}$. Além disso, a atividade muscular pode comportar-se de maneira diferente conforme a tipologia facial, atuando como fator contribuinte na etiologia da DTM ${ }^{37}$. Contudo, a literatura mostra que os hábitos orais deletérios representam fatores etiológicos freqüentemente associados à DTM ${ }^{19,38,39}$.

Faz-se necessário salientar que os instrumentos utilizados para diagnosticar DTM baseiam-se em questionários e/ou em exame clínico. Raramente os protocolos utilizados são padronizados, apresentando diferenças metodológicas, dificultando a comparação das pesquisas. Desta forma, os valores de prevalência variam de um estudo para outro, geralmente em virtude do método de investigação utilizado e de diferentes parâmetros de avaliação ${ }^{40}$.

Ressalta-se, também, a escassez de estudos sobre DTM em crianças utilizando metodologia padronizada em relação ao diagnóstico. Da mesma forma, poucos trabalhos abordam as pos- 
síveis etiologias da DTM nessa faixa etária, como aquelas relacionadas à oclusão, estado de conservação dos dentes, alterações de mastigação e deglutição, postura corporal, aspectos de ordem emocional, dentre outros. Tais informações auxiliarão na detecção e intervenção pelos profissionais da saúde, proporcionando a redução dos prejuízos relacionados.

\section{CONCLUSÃO}

Os resultados permitiram concluir que, das crianças avaliadas em um município de Rondônia, um terço (34\%) apresentou sinais de DTM, não havendo associação entre a DTM com a presença de hábitos orais deletérios nessa população.

\begin{abstract}
Purpose: to check the occurrence of temporomandibular disorder in children from Monte Negro/RO, correlating the findings to the presence of harmful oral habits. Methods: the study was conducted on 79 children aged form 6 to 11 -year old ( 41 girls and 38 boys). The presence of temporomandibular disorder was investigated and classified into articular, muscular and mixed. The presence of harmful oral habits involving sucking (baby bottle, pacifier, finger/thumb sucking) and chewing (nail biting, biting objects, biting the oral or labial mucosa, bruxism and clenching) was also found. Results: among the 79 investigated children, 27 (34.18\%) showed symptoms of temporomandibular disorder, with highest periodicity of articular disorder $(48.15 \%, n=13)$, followed by mixed $(33.33 \%, n=9)$ and muscular disorder $(18.52 \%, n=5)$. Harmful oral habits were found in $69.62 \%(n=55)$, mostly involving chewing habits $(39.91 \%, n=26)$ followed by sucking $(8.86 \%, n=7)$; some children showed both types of habits $(27.85 \%, n=22)$. The results did not demonstrate any association among harmful oral habits and temporomandibular disorder according to the chi-square test, regardless of the type of habit (chewing or suction). Conclusion: one third of children evaluated (34\%) presented symptoms of temporomandibular disorder, without any association between temporomandibular disorder and the presence of harmful oral habits.
\end{abstract}

KEYWORDS: Temporomandibular Joint; Child; Habits

\section{REFERÊNCIAS}

1. Zemlin WR. Articulação. In: Zemlin WR. Princípios de anatomia e fisiologia em fonoaudiologia. 2. ed. Porto Alegre: Artmed; 2000. p. 215-335.

2. Okeson JP. Diagnóstico diferencial e considerações sobre o tratamento das desordens temporomandibulares. In: Okeson JP. Dor orofacial: guia de avaliação, diagnóstico e tratamento. The American Academy of Orofacial Pain. Chicago: Quintessence; 1998. p. 113-84.

3. Bianchini EMG. Articulação temporomandibular e fonoaudiologia. In: Ferreira LP, Befi-Lopes DM, Limongi SCO, organizadores. Tratado de fonoaudiologia. São Paulo: Roca; 2004. p. 315-29.

4. Douglas CR. Fisiologia normal e patológica aplicada à odontologia e fonoaudiologia. In: Douglas CR. Patofisiologia oral: fisiologia normal e patológica aplicada à odontologia e fonoaudiologia. São Paulo: Pancast; 1998. p. 198-235.

5. Anelli-Bastos W, Oliveira MFR. Atuação fonoaudiológica na disfunção temporomandibular. In: Lopes
Filho O, organizador. Tratado de fonoaudiologia. 2. ed. Ribeirão Preto: Tecmedd; 2005. p. 755-65.

6. Thilander B, Rubio G, Pena L, Mayorga C. Prevalence of temporomandibular dysfunction and its association with malocclusion in children and adolescents: a epidemiologic study related to specified stages of dental development. Angle Orthod. 2002; 72(2):146-54.

7. Oliveira AS, Dias EM, Contato RG, Berzin F. Prevalence study of signs and symptoms of temporomandibular disorder in Brazilian college students. Braz Oral Res. 2006; 20(1):3-7.

8. Nilsson IM, List T, Drangsholt M. Incidence and temporal patterns of temporomandibular disorder pain among Swedish adolescents. J Orofac Pain. 2007; 21(2):127-32.

9. Almeida HS, Bomnarito S, Pino MR. Prevalência de sinais e sintomas de disfunção temporomandibular em mulheres. Odonto. 2003; 11(21):74-86.

10. Zhao YP, Ma XC. Temporomandibular disorders related pain interaction with age, sex and imaging changes of osteoarthrosis. Zhonghua Kou Qiang Yi Xue Za Zhi. 2006; 41(12):757-8. 
11. Barbosa CMR, Queluz DP, Barbosa JRA, Di Hipólito Júnior O. Correlação entre aparelho ortodôntico, sexo e presença de desordens temporomandibulares. J Bras Ortodon Ortop Facial. 2002; 7(39):185-92.

12. Bernal M, Tsamtsouris A. Signs and symptoms of temporomandibular joint dysfunction in 3 to 5 year old children. J Pedod. 1986; 10(2):127-40.

13. LeResche L, Mancl LA, Drangsholt MT, Huang $G$, Von Korff M. Predictors of onset of facial pain and temporomandibular disorders in early adolescence. Pain. 2007; 129(3):269-78.

14. Nilsson IM. Reliability, validity, incidence and impact of temporormandibular pain disorders in adolescents. Swed Dent J Suppl. 2007; 183:7-86.

15. Stegenga B, Schouten $\mathrm{H}$. Mandibular pain and movement disorders, focusing on the temporomandibular joint. Ned Tijdschr Tandheelkd. 2007; 114(1):41-6.

16. Bianchini EMG. Mastigação e ATM: avaliação e terapia. In: Marchesan IQ. Fundamentos em fonoaudiologia: aspectos clínicos da motricidade oral. 2. ed. Rio de Janeiro: Guanabara-Koogan; 2005. p. 45-58.

17. Pertes RA, Gross SG. Disfunções da articulação temporomandibular. In: Pertes RA, Gross SG. Tratamento clínico das disfunções temporomandibulares e da dor orofacial. 1. ed. São Paulo: Quintessence; 2005. p. 69-89.

18. Shiau YY, Peng CC, Wen SC, Lin LD, Wang JS, Lou KJ. The effects of masseter muscle pain on biting performance. J Oral Rehabil. 2003; 30(10):978-84.

19. Farsi N, Alamoudi N, Feteih R, El-Kateb $M$. Association between temporomandibular disorders and oral parafunctions in Saudi children. Odontostomatol Trop. 2004; 27(106):9-14.

20. Cheifetz AT, Osganian SK, Allred EN, Needleman HL. Prevalence of bruxism and associated correlates in children as reported by parents. J Dent Child. 2005; 72(2):67-73.

21. Castelo PM, Gavião MBD, Pereira LJ, Bonjardim LR. Relationship between oral parafunctional/ nutritive sucking habits and temporomandibular joint disfunction in primary dentition. Int J Pediatr Dent. 2005; 15:29-36.

22. Padamsee M, Ahlin JH, Ko CM, Tsamtsouris A. Functional disorders of the stomatognathic system: part II: a review. J Pedod. 1985; 10(1):1-21.

23. Soncini $F$, Dornelles $S$. Ocorrência de hábitos orais nocivos em crianças com 4 anos de idade de creches públicas no município de Porto Alegre (RS) Brasil. Pró-Fono. 2000; 12:103-8.
24. Tomita NE, Bijella VT, Franco L. Relação entre hábitos bucais e má oclusão em pré-escolares. Rev Saúde Publica. 2000; 34(3):299-303.

25. Souza NB, Vasconcelos TC. A Influência de hábitos orais como fator etiológico de mordida aberta anterior. Rev CEFAC. 2003; 5(3):235-40.

26. Figueiredo ES, Bianchini EMG, Crivello Júnior O. Hábitos parafuncionais em pacientes portadores de disfunção dolorosa da articulação temporomandibular (ATM). In: Marchesan IQ, Zorzi JL, Gomes ICD, organizadores. Tópicos em fonoaudiologia 1997/1998. São Paulo: Lovise; 1998. p. 213-32.

27. Sonnesen L, Bakke M, Solow B. Malocclusion traits and symptoms and signs of temporomandibular disorders in children with severe malocclusion. Eur J Orthod. 1998; 20(5):543-59.

28. Bonjardim LR, Gavião MB, Carmagnani FG, Pereira LJ, Castelo PM. Signs and symptoms of temporomandibular joint dysfunction in children with primary dentition. J Clin Pediatr Dent. 2003; 28(1):53-8.

29. Williamson EH. Temporomandibular dysfunction in pretreatment adolescent patients. Am J Orthod. 1977; 72(4):429-33.

30. Tuerlings V, Limme M. The prevalence of temporomandibular joint dysfunction in the mixed dentition. Eur J Orthod. 2004; 26(3):311-20.

31. Okeson JP. Diagnóstico das disfunções temporomandibulares. In: Okeson JP. Orofacial pain: guidelinef for assessment, diagnosis and management. The American Academy of Orofacial Pain. Chicago: Quintessence; 1996. p. 220-42.

32. Marklund S, Wänman A. Incidence and prevalence of temporomandibular joint pain and dysfunction: a one-year prospective study of university students. Acta Odontol Scand. 2007; 65(2):119-27.

33. Galvão ACUR, Menezes SFL, Nemr K. Correlação de hábitos orais deletérios entre crianças de 4 a 6 anos de escola pública e escola particular da cidade de Manaus - AM. Rev CEFAC. 2006; 8(3):328-36.

34. Pereira LF, Silva AMT, Cechella C. Ocorrência de hábitos orais viciosos e distúrbios fonoarticulatórios em indivíduos portadores de deglutição atípica. Pró-fono. 1998; 10(1):56-60.

35. Farsi NM. Symptoms and signs of temporomandibulardisorders and oral parafunctions among Saudi children. J Oral Rehabil. 2003; 30(12):1200-8.

36. Ramfjord SP, Ash MM. Occlusal adjustment of natural teeth. In: Ramfjord SP, Ash MM. Occlusion. Philadelphia: W. B. Saunders Company; 1966. p. 248-88. 
37. Cha BK, Kim CH, Baek SH. Skeletal sagittal and vertical facial types and electromyographic activity of the masticatory muscle. Angle Orthod. 2007; 77(3):463-70.

38. Molina OF. Tratamento multidisciplinar dos distúrbios craniomandibulares. In: Molina OF. Fisiopatologia craniomandibular (oclusão e ATM). 2. ed. São Paulo: Pancast; 1989. p. 595-677.
39. Tosato JP, Biasotto-Gonzalez DA, Gonzalez TO. Presence of temporomandibular joint discomfort related to pacifier use. Rev Bras Otorrinolaringol. 2005; 71(3): 365-8.

40. Deng YM, Fu MK, Hãgg U. Prevalence of temporomandibular joint disfunction (TMJD) in Chinese children and adolescents: a cross-sectional epidemiological study. Eur J Orthod. 1995; 17:305-9.

RECEBIDO EM: 13/06/2007

ACEITO EM: 08/11/2007

Endereço para correspondência:

Giédre Berretin-Felix

Departamento de Fonoaudiologia, Faculdade de Odontologia de Bauru / USP

Al. Dr. Octávio Pinheiro Brisolla 9-75

Bauru - SP

CEP: 17012-901

Tel: (14) 32358332 / (14) 32814773 / (14) 91126743

E-mail: gfelix@usp.br 